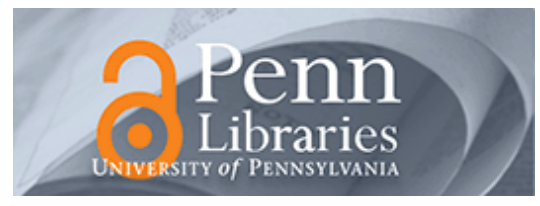

University of Pennsylvania

ScholarlyCommons

2000

\title{
Free Drainage of Aqueous Foams: Container Shape Effects on Capillarity and Vertical Gradients
}

\author{
A. Saint-Jalmes \\ University of California, Los Angeles
}

M. U. Vera

University of California, Los Angeles

Douglas J. Durian

University of Pennsylvania, djdurian@physics.upenn.edu

Follow this and additional works at: https://repository.upenn.edu/physics_papers

Part of the Physics Commons

\section{Recommended Citation}

Saint-Jalmes, A., Vera, M. U., \& Durian, D. J. (2000). Free Drainage of Aqueous Foams: Container Shape Effects on Capillarity and Vertical Gradients. Europhysics Letters, 50 (5), 695-701. http://dx.doi.org/ 10.1209/epl/i2000-00326-y

At the time of publication, author Douglas J. Durian was affiliated with University of California, Los Angeles. Currently, he is a faculty member at the Physics Department at the University of Pennsylvania.

This paper is posted at ScholarlyCommons. https://repository.upenn.edu/physics_papers/599

For more information, please contact repository@pobox.upenn.edu. 


\title{
Free Drainage of Aqueous Foams: Container Shape Effects on Capillarity and Vertical Gradients
}

\author{
Abstract \\ The standard drainage equation applies only to foam columns of constant cross-sectional area. Here, we \\ generalize to include the effects of arbitrary container shape and develop an exact solution for an \\ exponential, "Eiffel Tower", sample. This geometry largely eliminates vertical wetness gradients, and \\ hence capillary effects, and should permit a clean test of dissipation mechanisms. Agreement with \\ experiment is not achieved at late times, however, highlighting the importance of both boundary \\ conditions and coarsening.

\section{Disciplines} \\ Physical Sciences and Mathematics | Physics

\section{Comments} \\ At the time of publication, author Douglas J. Durian was affiliated with University of California, Los \\ Angeles. Currently, he is a faculty member at the Physics Department at the University of Pennsylvania.
}


Europhys. Lett., 50 (5), pp. 695-701 (2000)

\title{
Free drainage of aqueous foams: Container shape effects on capillarity and vertical gradients
}

\author{
A. Saint-Jalmes, M. U. Vera and D. J. Durian \\ UCLA Department of Physics and Astronomy - Los Angeles, CA 90095-154\%, USA
}

(received 9 August 1999; accepted in final form 7 April 2000)

PACS. 82.70.Rr - Aerosols and foams.

PACS. 47.60.+i - Flows in ducts, channels, nozzles, and conduits.

PACS. 47.55. Mh - Flows through porous media.

\begin{abstract}
The standard drainage equation applies only to foam columns of constant crosssectional area. Here, we generalize to include the effects of arbitrary container shape and develop an exact solution for an exponential, "Eiffel Tower", sample. This geometry largely eliminates vertical wetness gradients, and hence capillary effects, and should permit a clean test of dissipation mechanisms. Agreement with experiment is not achieved at late times, however, highlighting the importance of both boundary conditions and coarsening.
\end{abstract}

Introduction. - Free drainage is the unavoidable fate of aqueous foams under Earth's gravity $[1,2]$. Because of the density mismatch between gas and liquid, the bubbles rise and collect at the top, while the liquid falls through the random network of plateau borders and accumulates at the bottom. This behavior can be understood in general terms via a nonlinear partial differential "drainage equation" that expresses liquid conservation as flow proceeds in response to gravity, capillary, and viscous forces [3-5]. However, in spite of its obvious importance for applications, and its apparent simplicity, the problem of free drainage in foams is not well understood yet [6]. One reason is that analytic predictions of the full time dependence cannot be achieved unless capillary terms, the most egregious nonlinearity, are dropped from the drainage equation [7]. This cannot be a valid approximation for short columns, at late times, or even at early times for dry foams [6]. Another reason is that the mechanism of viscous dissipation is not clearly established; shear flow may occur within the plateau borders at which three films meet [3], or only within the vertices at which four plateau borders meet [8]. Only recently have experiments been carried out systematically as a function of uniform initial liquid fraction and sample height [6]. Even then it was not possible to isolate and identify the individual effects of capillarity, dissipation, and potentially coarsening, because of their strong nonlinear couplings.

In this paper we attack the free-drainage problem with an experiment designed to minimize capillary effects and, thereby, to isolate and identify dissipation mechanisms. This is accomplished by altering the sample geometry in such a way that drainage may proceed without vertical gradients in liquid fraction. Since the standard drainage equation applies only to straight columns of constant cross-section, we begin by generalizing to account for arbitrary container shape. We then develop an exact solution for special containers that flare out exponentially towards the bottom, much like the Eiffel tower. At each height, we predict, more liquid can be passed down than received from above, so that the foam becomes uniformly drier with time. Finally, we compare with experiments using specially constructed "Eiffel 
towers" of two different flaring lengths. At early times we find good agreement; at later times, however, discrepancies arise due to neglect of both boundary conditions and coarsening.

Generalized drainage equation. - The basic goal of all free-drainage models is to determine the time evolution of the liquid content vs. depth, $z$, into the foam. For fairly dry foams, the liquid is distributed within three distinct structures: the flat soap films that separate two neighboring bubbles; the long Plateau Borders (PBs), of scalloped-triangular cross-section, at which three soap films meet; and the scalloped-tetrahedral vertices at which four PBs meet [1-3]. Ignoring numerical factors, the liquid fraction is thus $\varepsilon \sim\left(R^{2} l+R r^{2}+r^{3}\right) / R^{3}$, where $R$ is the sphere-equivalent bubble radius, $r$ is the radius of curvature of both the PBs and vertices, and $l$ is the thickness of the soap films. Since $l \ll r \ll R$ typically holds, one gets $\varepsilon \sim(r / R)^{2}$, meaning that the liquid resides almost exclusively in the random network of PBs. Within one PB, the flow speed may be deduced by the balance of gravity, viscosity and capillarity:

$$
u=u_{0} \varepsilon^{m}\left(1-\sqrt{\frac{\varepsilon_{\mathrm{c}}}{\varepsilon}} \frac{\xi}{\varepsilon} \frac{\partial \varepsilon}{\partial z}\right) .
$$

The value of $m$ is set by the nature of viscous dissipation, being 1 if shear flow is primarily in the plateau borders [3] and $1 / 2$ if primarily in the vertices [8]. The two quantities that carry all dimensions are a maximum characteristic flow speed, $u_{0}$, and a capillary rise scale, $\xi$, set, respectively, by the competition between dissipation and capillary forces against gravity. These must scale as $u_{0} \sim \rho g R^{2} / \eta$ and $\xi \sim \gamma / \rho g R$; the precise numerical coefficients depend on details of the film $/ \mathrm{PB} /$ vertex geometry and the dissipation mechanism, but are not well known [6]. And finally, $\varepsilon_{\mathrm{c}}=1-0.635$ is a critical liquid fraction where the bubbles become randomly close-packed spheres. This way of writing the flow speed emphasizes the role of capillarity through the gradient term. According to eq. (1), for any dissipation mechanism and for any sample geometry of height $h$, free drainage eventually stops and $u \rightarrow 0$ when the liquid fraction reaches a final capillary profile of $\varepsilon(z)=\varepsilon_{\mathrm{c}} /[1+(h-z) / 2 \xi]^{2}[9-12]$; note that at the bottom $\varepsilon(h)=\varepsilon_{\mathrm{c}}$ and $\varepsilon /\left.(\partial \varepsilon / \partial z)\right|_{h}=\xi$. Thus $\xi$ may be defined operationally as the extrapolation length of the equilibrium capillary profile. And similarly, if the boundary is drier than $\varepsilon_{\mathrm{c}}$, eq. (1) implies a larger extrapolation length is required for a no-flow boundary condition: $\varepsilon /\left.(\partial \varepsilon / \partial z)\right|_{\text {boundary }}=\left.\xi \sqrt{\varepsilon_{\mathrm{c}} / \varepsilon}\right|_{\text {boundary }}$.

Assuming that the flow is only downwards (in the $+z$ direction) according to eq. (1), it is straighforward to write a drainage equation for the behavior of $\varepsilon(z, t)$ applicable for any container shape. We simply require conservation for the total amount of liquid at depth $z$, which is proportional to the product of liquid fraction, $\varepsilon$, and the cross-sectional area, $A(z)$, of the container at $z$. After separating out the derivatives of $A$, then dividing by $A$, the continuity equation becomes

$$
\left[\frac{\partial \varepsilon}{\partial t}+\frac{\partial(u \varepsilon)}{\partial z}\right]+\left[\frac{u \varepsilon}{A} \frac{\mathrm{d} A}{\mathrm{~d} z}\right]=0,
$$

where $u$ is given by eq.(1). The first term in brackets represents the usual drainage equation. The $\mathrm{d} A / \mathrm{d} z$ term represents the effects of container shape. It is straightforward to modify eqs. (1)-(2) for three-dimensional problems, such as lateral flow in free drainage or radial flow in a spinning container. To our knowledge, the only prior investigation into container shape is ref. [10], where the total liquid content was compared with that in the remaining capillary profile to determine whether or not leakage could occur; dynamics were not considered.

Uniform column predictions. - For a column with constant cross-section, the complete free-drainage problem may be solved only if the capillary term is dropped [13]. In this ap- 
proximation, the liquid fraction decreases from an initial value of $\varepsilon_{0}$ according to [6]

$$
\varepsilon(z, t)=\varepsilon_{0}\left\{\begin{array}{ll}
(z / v t)^{1 / m}, & z \leq v t \\
1, & z \geq v t
\end{array} \quad \text { with } v=u_{0}(m+1) \varepsilon_{0}^{m} .\right.
$$

There is thus a drying front that travels from the top of the sample downwards at constant speed $v$. Below it, the foam maintains the same initial wetness as liquid leaks out at a constant rate. Once the front hits the bottom of the sample, the rate of drainage progressively decreases until no liquid remains. The amount of drained liquid vs. time may be found by integration:

$$
V(t) / V_{\mathrm{f}}= \begin{cases}\frac{1}{m+1}(v t / h), & v t \leq h, \\ 1-\frac{m}{m+1}(h / v t)^{1 / m}, & v t \geq h,\end{cases}
$$

where the final value $V_{\mathrm{f}}$ equals the total amount of liquid within the sample at time zero.

Eiffel tower predictions. - Even though approximation is necessary for a uniform column, exact analytic solution of the drainage equation can be achieved if the container shape flares out exponentially towards the bottom, like the Eiffel tower. For $A(z)=A_{0} \exp \left[z / z_{0}\right]$, it is simple to verify that a solution of eq. (2) exists where the capillary term, $\partial \varepsilon / \partial z$, vanishes and where the liquid fraction thus decreases uniformly across the entire height of the sample:

$$
\varepsilon(z, t)=\varepsilon_{0}\left(1+t / t_{0}\right)^{-1 / m}, \quad \text { with } t_{0}=z_{0} /\left(u_{0} m \varepsilon_{0}^{m}\right) .
$$

The corresponding volume of drained liquid is simply

$$
V(t) / V_{\mathrm{f}}=1-\left(1+t / t_{0}\right)^{-1 / m} .
$$

At short times, both $\varepsilon(z, t)$ and $V(t)$ vary linearly with $t$. At long times, they exhibit power law asymptotes with an exponent that depends on dissipation mechanism. Note that neither eq. (3) nor eq. (5) satisfies an extrapolation or $\varepsilon \rightarrow \varepsilon_{\mathrm{c}}$ boundary condition at the bottom, $z=h$. Therefore, these predictions neglect a possible delay in onset of leakage at short times and the final wetness profile at long times. Such capillary effects may be especially important for dry foams and short columns, but can be handled only by numerical solution.

Experiments. - Foams are produced using a turbulent mixing technique described earlier [6]. It rapidly provides large volumes of foam, enough to fill our tanks in less than 1 minute, with any initial liquid fraction desired in the range $3 \%<\varepsilon_{0}<45 \%$. Here the gas is $\mathrm{N}_{2}$, and the liquid is water with $0.4 \% \alpha$-olefinsulfonate by weight. The bubble size distribution is slightly polydisperse with an average diameter of about $110 \mu \mathrm{m}$, independent of $\varepsilon_{0}$. The polydisersity does not noticeably change with either drainage or coarsening. Also as before [6], the viscous flow speed and capillary length scales are, respectively, of order $u_{0} \approx 0.05 \mathrm{~cm} / \mathrm{s}$ and $\xi \approx 4 \mathrm{~cm}$. At time zero such foams are flowed directly into one of three different containers, all with the same thickness of $1.25 \mathrm{~cm}$ and the same height of $h=70 \mathrm{~cm}$. The first is rectangular, for reference, with a constant width of $25 \mathrm{~cm}$. The other two have the same $25 \mathrm{~cm}$ width at the bottom, but flare out exponentially from a smaller width at the top according to either $z_{0}=25$ or $46 \mathrm{~cm}$. These "Eiffel towers" can also be inverted for additional shape variation (though eqs. (5)-(6) apply only for $z_{0}>0$ ).

The importance of container shape may be demonstrated by differences in drainage curve data, $V(t) / V_{\mathrm{f}}$ vs. $t$, for foams with the same initial liquid fraction of $\varepsilon_{0}=0.36$ (close to $\left.\varepsilon_{\mathrm{c}}\right)$. The results in fig. 1(a) show that drainage is initially most rapid in the Eiffel tower with $z_{0}=25 \mathrm{~cm}$; it is initially slowest when this tank is inverted. For $z_{0}=46 \mathrm{~cm}$ the shape variation 


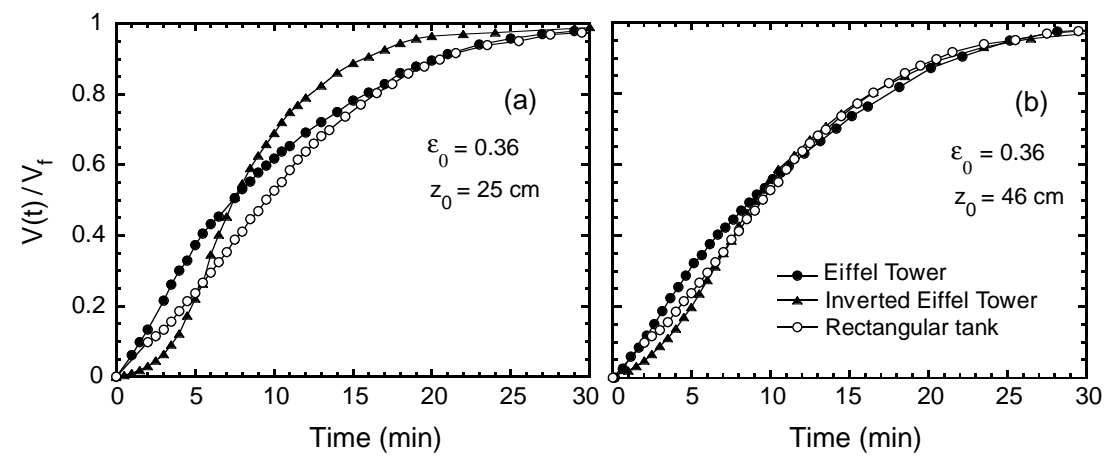

Fig. 1 - Drainage curves of a foam with $\varepsilon_{0}=0.36$, for a rectangular tank and two Eiffel towers $\left(z_{0}=\right.$ 25 and $46 \mathrm{~cm}$ ) in both upright and inverted configurations.

is more gentle, and differences are correspondingly reduced; results in fig.1(b) for both upright and inverted configurations are closer to those for the rectangular tank. At late times the final drainage behavior $V(t) / V_{\mathrm{f}} \rightarrow 1$ is fastest, surprisingly, in the inverted Eiffel tower. One might have thought that the upright configuration should drain more rapidly, because at the bottom there is both more liquid at time zero and more PBs connected in parallel. While this may help at short times, the inverted configuration ultimately wins because the draining liquid is channelled into progressively fewer and fewer PBs, which then become thicker and allow liquid to pass through with even less viscous resistance. In fact, we observe that the wetness can even exceed $\varepsilon_{\mathrm{c}}$ over an extended region near the bottom, as is evident from a rapid turbulent-like seething of the bubbles. It is amusing to demonstrate this phenomenon by vigorously shaking soapy water in a flask and allowing it to drain in either upright or inverted configurations.

Before comparing drainage curves with eqs. (4) and (6), we first investigate whether there exists a domain of validity where neither wetness gradients nor coarsening are important. This is done by uniformly illuminating the sample and measuring the diffusely transmitted light vs. position with a CCD camera. This signal increases monotonically as the bubbles become drier via drainage, or larger via coarsening, though the precise calibration is not yet known. Results at several foam ages are shown in fig. 2 for different container shapes and initial degrees of wetness. For a wet foam in the rectangular tank, fig. 2(a), we can see the expected free-drainage behavior. Namely, a dryness front propagates downward while the rest of the foam maintains the same initial wetness; capillarity serves to round the front and to create the final wetness gradient. For a wet foam in the $z_{0}=25 \mathrm{~cm}$ Eiffel tower, fig. 2(b), by contrast, we observe that the initial decrease in liquid content is uniform across the entire height of the sample. In accord with the prediction of eq. (5), there are no wetness gradients. At longer times, however, a $z$-dependent capillary profile eventually develops, beginning first at the bottom of the tank where $\varepsilon(h)=\varepsilon_{\mathrm{c}}$ is always maintained. For the $z_{0}=46 \mathrm{~cm}$ Eiffel tower, transmission data (not shown) reveal a drying front at the top and a uniform drying at the bottom. This hybrid behavior arises because the height is not great enough compared to $z_{0}$ so that the width at the top becomes small; Eiffel towers must not be truncated if eqs. (5)(6) are to apply. For the dry foams, figs. 2(c)-(d), we observe qualitatively different behavior. Here, the total initial volume of liquid in the foam (proportional to either $\varepsilon_{0} h$ or $\varepsilon_{0} z_{0}$ ) is not great compared to that needed for the final capillary profile $\left(\varepsilon_{\mathrm{c}} \xi\right)$. Thus, the liquid can leak out only as coarsening proceeds and the capillary length decreases in inverse proportion to the average bubble size. In both rectangular and exponential tanks, the liquid content decreases 

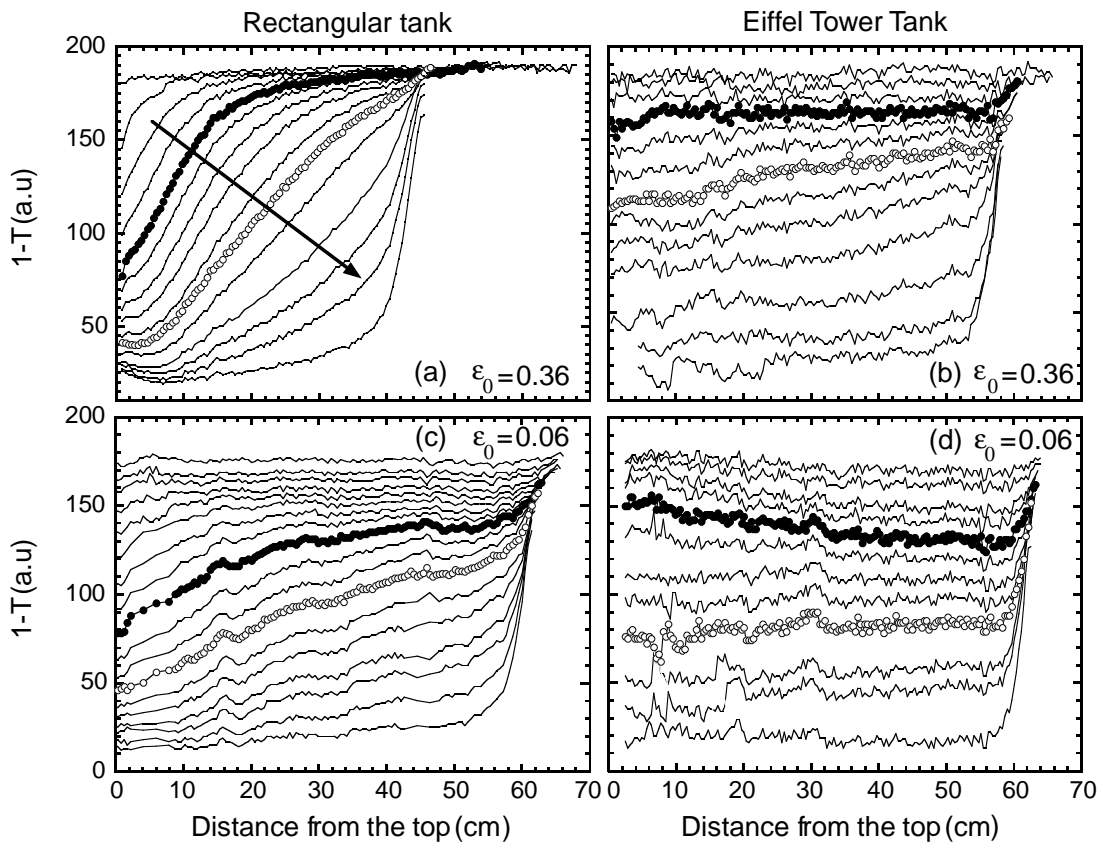

Fig. 2 - Evolution of diffusely transmitted light (arbitrary units) vs. depth for four different cases: wet $\varepsilon_{0}=0.36$ and dry $\varepsilon_{0}=0.06$ foams in rectangular and $z_{0}=25 \mathrm{~cm}$ Eiffel tower tanks, as labeled. In each plot, the curves at which $1 / 2$ and $3 / 4$ of all liquid has drained are highlighted. The arrow indicates the direction of time. In minutes, the specific time sequences are (a) $0.5,3.25,5,7.25,9.5$, $11,12.5,13.25,14,15,17.75,20,24.25,27,29.75$; (b) $0.5,3.25,5,7.5,9.5,12,14,15.75,17,19,23.25$, 27,31 ; (c) $0.5,3.5,4.75,6,7.75,8.5,9.5,10.25,11,12,13.75,15.75,18,20.25,22.5,24,27,32$; and (d) $0.5,2.25,6,7.25,8.75,9.75,12,13.5,15,20.5,23.75,31$.

uniformly at short times, becoming slightly drier at the top and bottom, respectively, before developing a recognizable capillary profile.

According to fig. 2, quantitative comparison of the drainage curves with analytic predictions may only be made for the wet $\varepsilon_{0}=0.36$ foam at short times in the rectangular and $z_{0}=25 \mathrm{~cm}$ Eiffel tower tanks. Equations (4) and (6) predict that $V(t) / V_{\mathrm{f}}$ should initially grow in proportion to time with slopes of $u_{0} \varepsilon_{0}^{m} / h$ and $u_{0} \varepsilon_{0}^{m} / z_{0}$, respectively. As can be seen by the dashed lines in fig. 3(a)-(b), the volume of drained liquid indeed grows linearly at short times, with slopes of $1 /(20 \mathrm{~min})$ and $1 /(12 \mathrm{~min})$, respectively. The ratio of these slopes is 0.6 , which is somewhat larger than the expectation $z_{0} / h=0.4$. One possible source for this discrepancy is that the clear division of the foam microstructure into films + plateau borders + vertices, as assumed in the drainage equations, is valid only for rather dry foams $\varepsilon<0.1[5]$.

At longer times, we may investigate the breakdown of theory by plotting the full predictions using the initial slopes already extracted and quoted above. These comparisons are shown in fig. 3 for both $m=1$ (PB dissipation) and $m=1 / 2$ (vertex dissipation). Noticeable deviation sets in by the time $V(t) / V_{\mathrm{f}}$ rises to $1 / 2$, which corresponds reasonably well with the onset of a capillary profile in the diffuse transmission data of fig. 2 . This effect could be captured by numerical solution of the drainage equation using the proper boundary conditions. However, capillarity pushes liquid from wetter to drier regions and should hence slow down the leakage; therefore, it cannot fully account for our drainage curve data, which rise faster than expected 


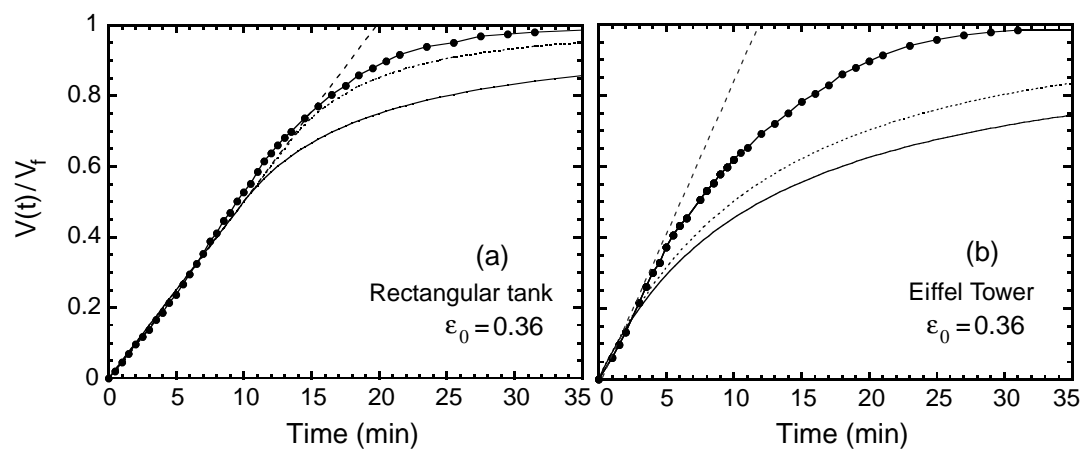

Fig. 3 - Drainage curves for wet foams, $\varepsilon_{0}=0.36$, in rectangular and $z_{0}=25 \mathrm{~cm}$ Eiffel tower tanks, as labeled. Dashed lines show the initial linear behavior; the dotted and solid curves continue from the linear growth according to the full predictions with, respectively, $m=1 / 2$ for vertex-dominated dissipation and $m=1$ for PBs-dominated dissipation.

towards complete drainage at late times. This behavior, we believe, is due to coarsening. As the foam drains, there is less liquid between neighboring bubbles and the rate of gas migration thus increases. As the average bubble size grows, the viscous flow speed increases according to eq. (1) and the rate of drainage thus increases even further. This scenario is revealed simply by visual inspection of surface bubbles $v s$. time: in the middle of the tank, the average bubble size grows to about $1 \mathrm{~mm}$ after 30 minutes. It can also be seen in the relatively large change in diffuse transmission, fig. (2), at late times after about 3/4 of the liquid has drained; there, the light transmission increases more because the average bubble size is growing rather than because the liquid content is decreasing. Coarsening also explains two other features of our drainage curves. First, the onset of discrepancy is not as severe for the straight column since the lower portion maintains its wetness longer and hence coarsens more slowly. Second, the final approach $V(t) / V_{\mathrm{f}} \rightarrow 1$ is achieved via evolution of the capillary profile by coarsening, and is hence almost identical for both tanks (the remaining liquid is proportional to $\varepsilon_{\mathrm{c}} \xi$ and decays as $1 / R \sim 1 / \sqrt{t}$ according to the usual [14] growth law).

Conclusions and perspectives. - We have proposed a generalized version of the drainage equation that accounts for arbitrary container shapes. For the special case of free drainage in an exponential "Eiffel Tower", we have solved it analytically neglecting only capillarity in the boundary conditions. By contrast with a straight column, there is no drying front and vertical gradients are suppressed at early times. Experiments confirm this picture and provide time scales for the initial growth that are in good accord with those for rectangular columns. However, at late times and for dry foams, serious discrepancies arise due to the neglect of capillarity and coarsening. The former may be handled by numerically implementing the proper boundary conditions. It may be possible to handle the latter via the bubble-size dependence of $u$ in eq. (1). Since it is unavoidable that foams coarsen more rapidly as they become drier, this is important for an understanding of the free-drainage problem in general.

$$
* * *
$$

We thank S. Hilgenfeldt, S. A. Koehler and H. A. Stone for helpful discussions and comments. This work was supported by NASA through grant NAG3-1419 and by NSF through grant DMR-9623567. 


\section{REFERENCES}

[1] Bikerman J.J, Foams (Springer-Verlag, New York) 1973.

[2] Prud'homme R. K. and Khan S. A. (Editors), Foams: Theory, Measurement, and Application, Surf. Sci. Ser., Vol. 57 (Marcel Dekker, New York) 1996.

[3] Weaire D., Hutzler S., Verbist G. and Peters E., Adv. Chem. Phys., 102 (1997) 315.

[4] Bhakta A. and Ruckenstein E., Adv. Colloid Interface Sci., 70 (1997) 1.

[5] Koehler S. A., Stone H. A., Brenner M. P. and Eggers J., Phys. Rev. E, 58 (1998) 2097.

[6] Saint-Jalmes A., Vera M. U. and Durian D. J., Eur. Phys. J. B, 12 (1999) 67.

[7] As shown in ref. [5], exact similarity solutions do exist. While useful in certain limits, these cannot predict the flux of drained liquid out from the sample.

[8] Koehler S. A., Hilgenfeldt S. and Stone H. A., Phys. Rev. Lett., 82 (1999) 4232.

[9] Princen H. M. and Kiss A. D., Langmuir, 3 (1987) 36.

[10] Princen H. M., J. Colloid Interface Sci., 134 (1990) 188.

[11] Bhakta A. and Ruckenstein E., Langmuir, 11 (1995) 1486.

[12] Verbist G., Weaire D. and Kraynik A. M., J. Phys. Condens. Matter, 8 (1996) 3715.

[13] KrayniK, A. M., unpublished internal report SANDIA 83-0844 (1983)

[14] Stavans, J., Rep. Prog. Phys., 56 (1993) 733. 